Supporting Information

\title{
Fluorescent polypropylene nanoplastics for studying uptake, biodistribution, and excretion in zebrafish embryos
}

Wang Sik Lee, ${ }^{1}$ Hyunjung Kim, ${ }^{1}$ Yugyeong Sim,,${ }^{1,2}$ Taejoon Kang, ${ }^{3}$ and Jinyoung Jeong ${ }^{1,2 *}$ ${ }^{1}$ Environmental Disease Research Center, Korea Research Institute of Bioscience and Biotechnology (KRIBB), 125 Gwahak-ro, Yuseong-gu, Daejeon 34141, Republic of Korea ${ }^{2}$ Department of Nanobiotechnology, KRIBB School of Biotechnology, University of Science and Technology (UST), 217 Gajeong-ro, Yuseong-gu, Daejeon 34113, Republic of Korea ${ }^{3}$ Bionanotechnology Research Center, Korea Research Institute of Bioscience and Biotechnology (KRIBB), 125 Gwahak-ro, Yuseong-gu, Daejeon 34141, Republic of Korea Corresponding author: Jinyoung Jeong (jyjeong @kribb.re.kr) 


\section{Experimental section}

\section{Quantitative analysis of R-PPNPs in zebrafish embryos (ZFEs)}

We employed the fluorescence intensity of RBITC-labeled PPNPs (R-PPNPs) in a quantitative relationship model to determine the amount of R-PPNPs remaining in ZFEs. To do so, ZFEs were treated with 50 ppm R-PPNPs at $72 \mathrm{hpf}$ for $24 \mathrm{~h}$ and then washed 3 times with fresh EW and fixed with 4\% paraformaldehyde at 96 hpf and 120 hpf. Each ZFE was homogenized, and the fluorescence intensity was measured. The amounts of R-PPNPs in ZFEs were calculated by adjusting the standard curve. 
Table S1. The yield of PPNPs production by NIPS method

\begin{tabular}{cccccc}
\hline \multirow{2}{*}{ Number } & \multicolumn{3}{c}{ Mass $(\mathbf{m g})$} & Yield (\%) & Average \\
\cline { 2 - 3 } & In & Out & & \\
2 & 130 & 100 & $76.9 \%$ & \\
3 & 130 & 100 & $76.9 \%$ & $84.1 \%$ \\
4 & 260 & 230 & $88.5 \%$ & \\
\hline
\end{tabular}


Table S2. DSC melting results of PP pellets and PPNPs

\begin{tabular}{cccccc}
\hline Samples & $\mathbf{T c}\left({ }^{\circ} \mathbf{C}\right)$ & $\mathbf{T}_{\text {m-low }}\left({ }^{\circ} \mathbf{C}\right)$ & $\mathbf{T}_{\text {m-high }}\left({ }^{\circ} \mathrm{C}\right)$ & $\Delta \mathbf{H}_{\mathbf{f}}(\mathbf{J} / \mathbf{g})$ & $\mathbf{X}_{\text {DSC }}(\mathbf{\%})$ \\
\hline PP pellets & 115.40 & 147.53 & 156.52 & 102.39 & 49.44 \\
PPNPs & 120.87 & 149.54 & 156.64 & 105.31 & 50.85 \\
\hline
\end{tabular}


Table S3. Mortality and deformity of PPNPs and R-PPNPs-treated ZFEs $(\mathrm{P}>0.05)$

\begin{tabular}{lccccc}
\hline \multicolumn{2}{c}{ Conc. (ppm) } & \multicolumn{2}{c}{ Mortality rate (\%) } & \multicolumn{2}{c}{ Deformity rate (\%) } \\
& 0 & $13.33 \pm 5.77$ & 0.00 & $3.33 \pm 5.77$ & 0.00 \\
\hline \multirow{2}{*}{ R-PPNPs } & 50 & $13.33 \pm 5.77$ & 0.00 & $6.66 \pm 5.77$ & 0.00 \\
& 0 & $6.66 \pm 5.77$ & 0.00 & 0.00 & 0.00 \\
PPNPs & 50 & 0.00 & 0.00 & $3.33 \pm 5.77$ & 0.00 \\
\hline
\end{tabular}



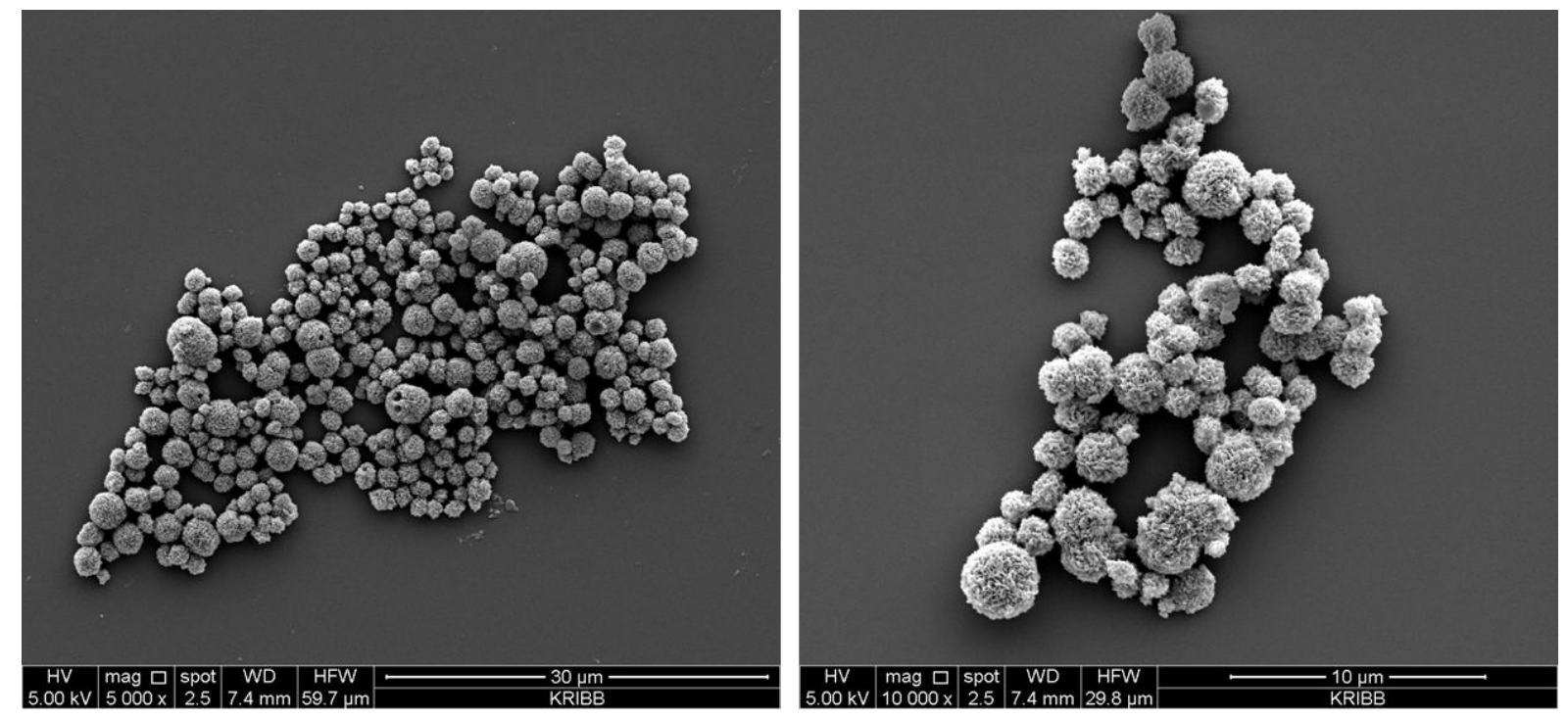

Figure S1. SEM images of PPNPs prepared by NIPS method using distilled water as a nonsolvent.
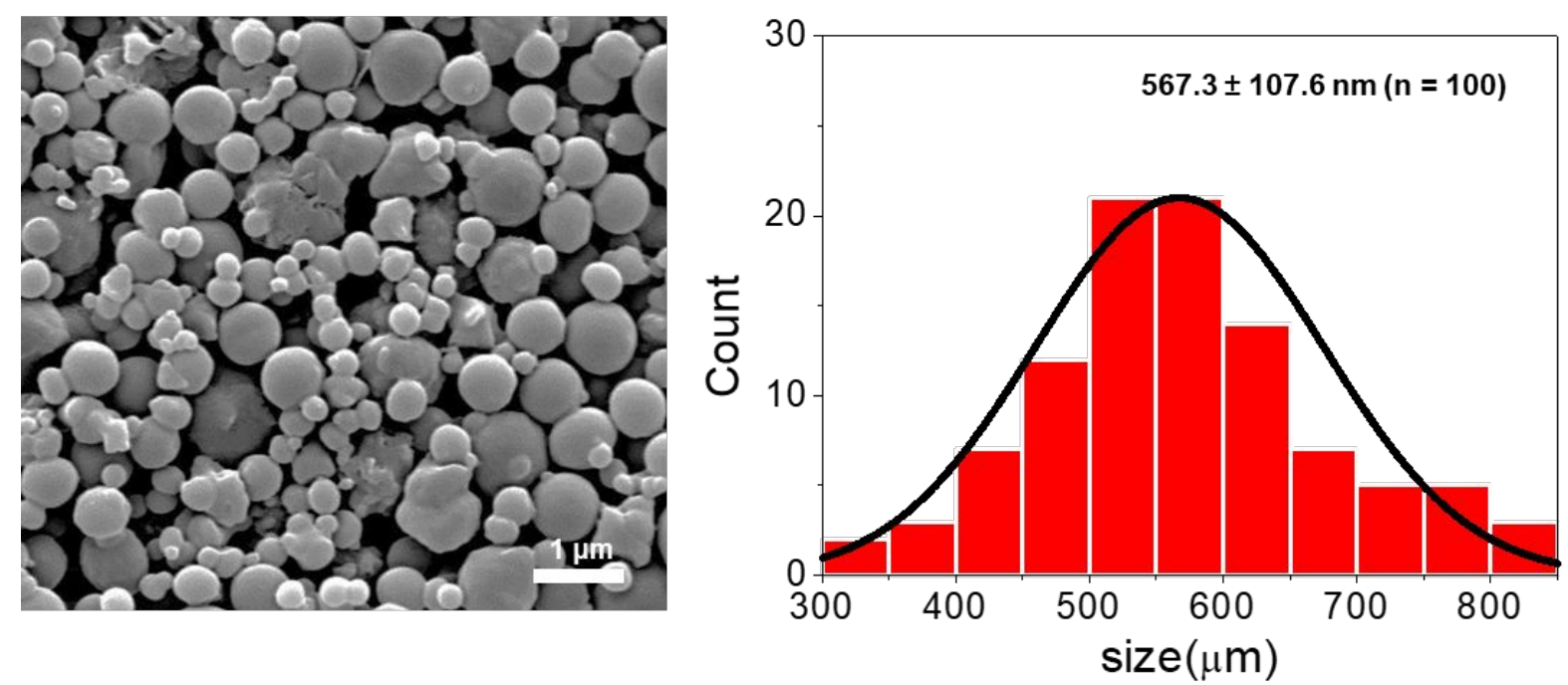

Figure. S2. SEM images of R-PPNPs and its size distribution. 


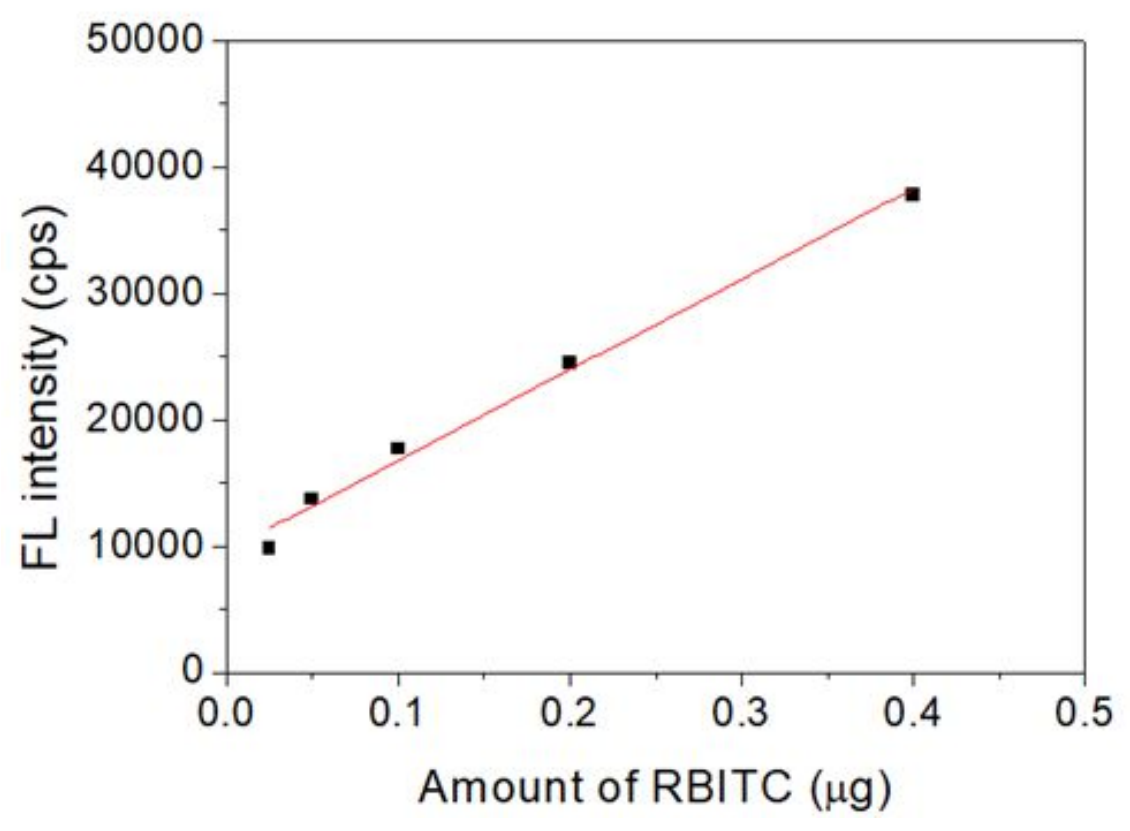

Figure S3. Standard curve for fluorescence intensity of RBITC by concentration. 

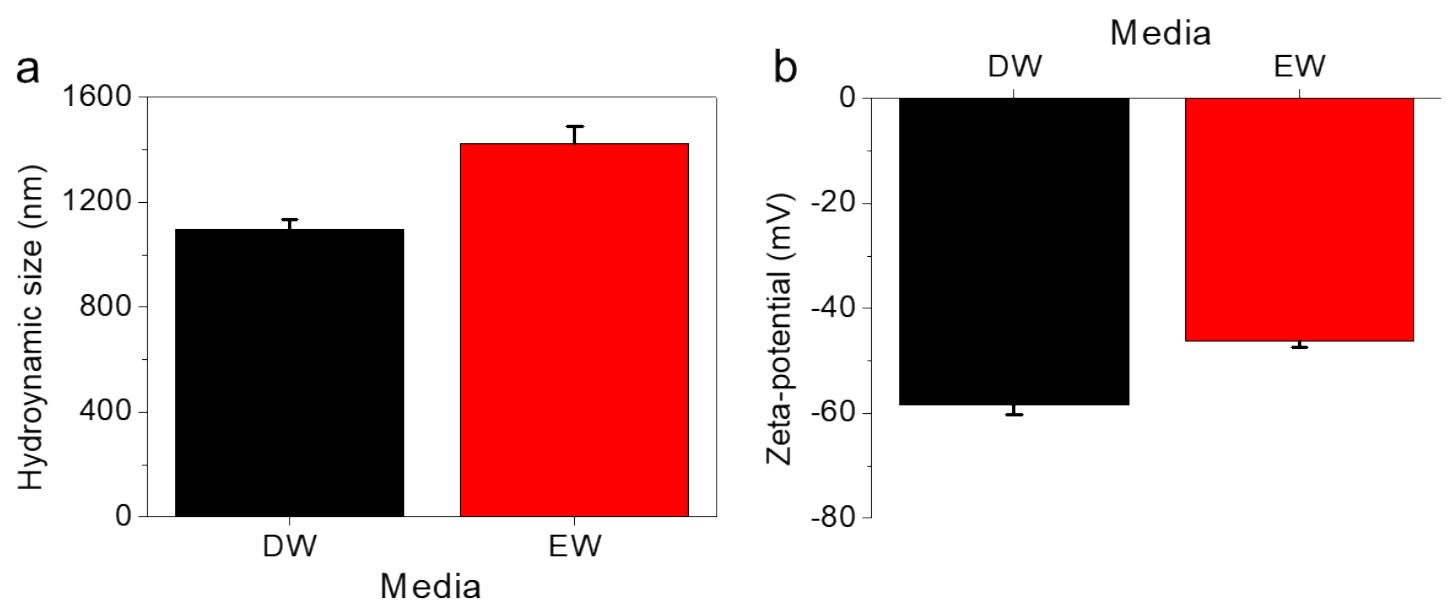

Figure S4. (a) Hydrodynamic size and (b) Zeta-potential values of PPNPs by different media (DW and EW) 


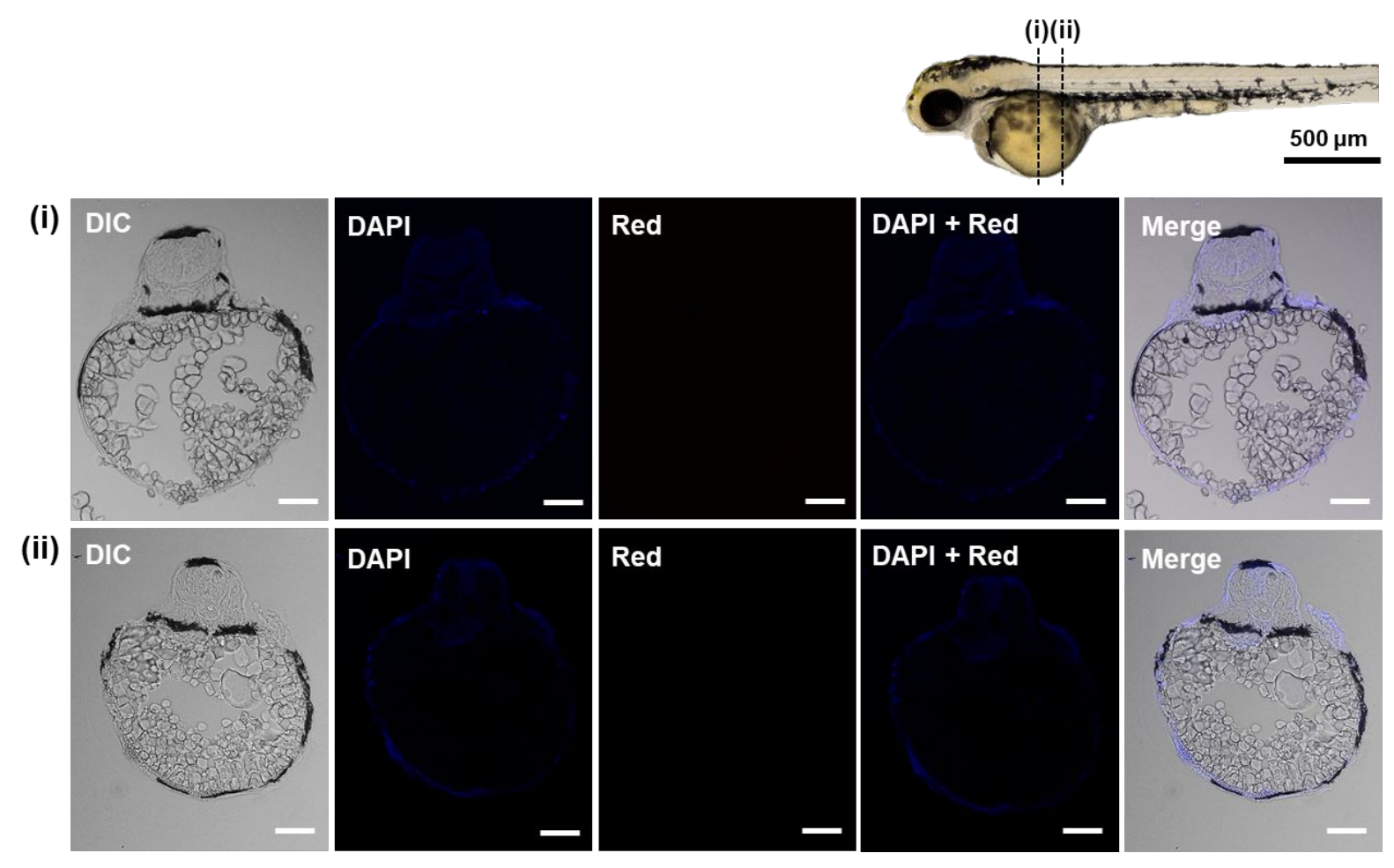

Figure S5. Cross-sectional images of the yolk sac (The point of dash line in top image i , ii ) in ZFEs at $48 \mathrm{hpf}$ after R-PPNPs exposure at $24 \mathrm{hpf}$ for $24 \mathrm{~h}$ with three different filters such as DIC, DAPI, and Red. 


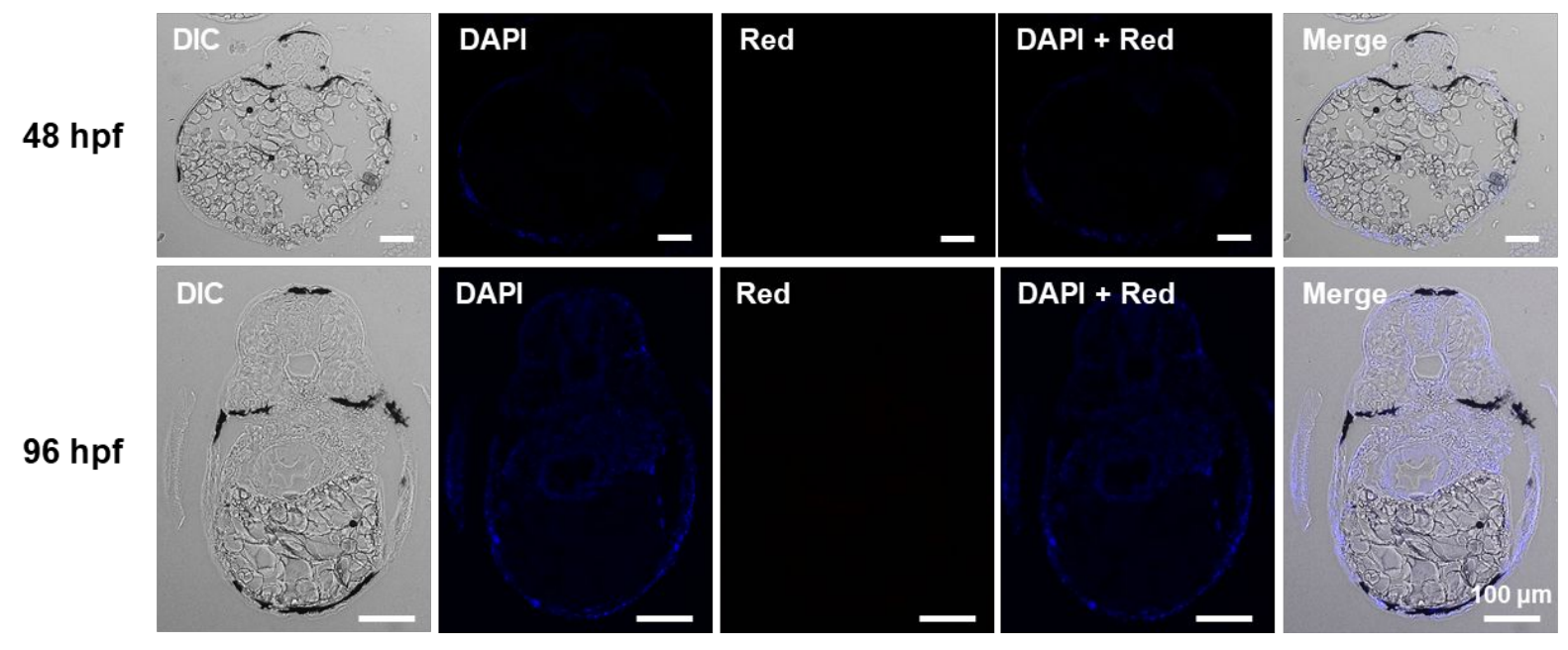

Figure S6. Cross-sectional images of control ZFEs at $48 \mathrm{hpf}$ and $96 \mathrm{hpf}$ with three different filters such as DIC, DAPI, and Red. 


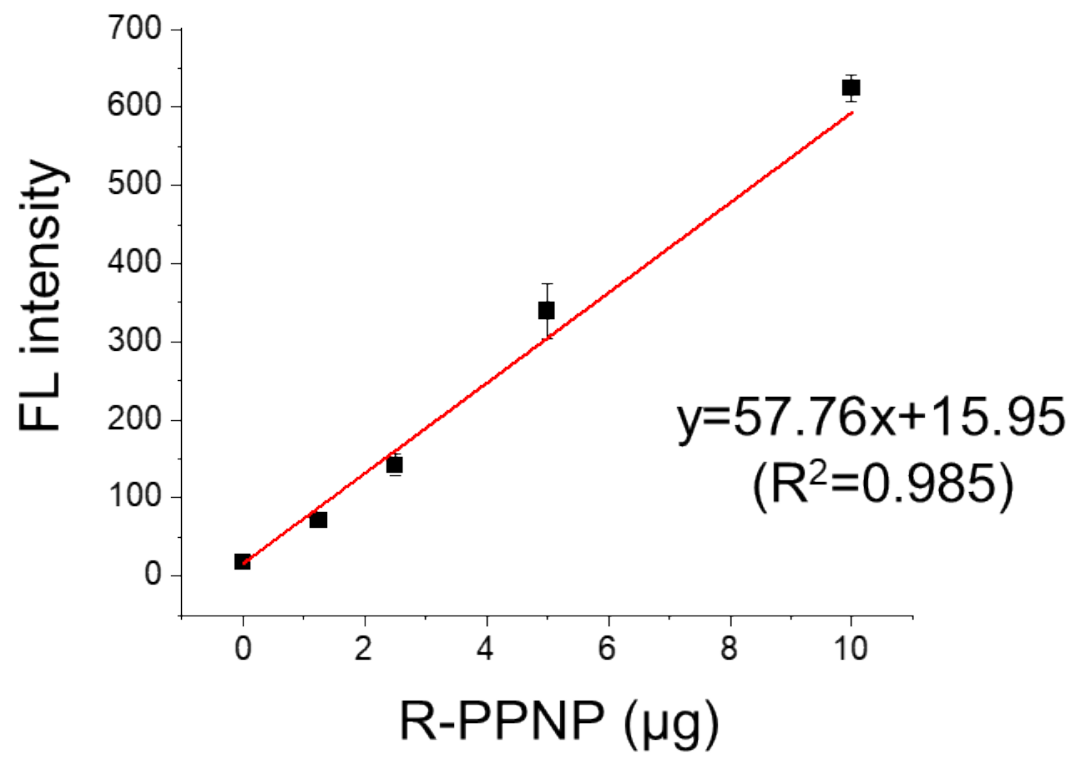

\begin{tabular}{ccc}
\hline$(\mathrm{n}=5)$ & $96 \mathrm{hpf}$ & $120 \mathrm{hpf}$ \\
\hline Control & $0.00 \pm 0.05 \mu \mathrm{g} /$ individual & $0.00 \pm 0.00 \mu \mathrm{g} /$ individual \\
R-PPNP & $1.01 \pm 0.09 \mu \mathrm{g} /$ individual & $0.46 \pm 0.10 \mu \mathrm{g} /$ individual \\
\hline
\end{tabular}

Figure S7. Standard curve and amount of R-PPNPs measured by fluorescent microplate reader (Cytation 5, BioTek, USA) under excitation/emission at 540/580 nm. 